\title{
Shared and item-specific information in memory for event descriptions
}

\author{
R. REED HUNT, JAYNE ANN AUSLEY \\ University of North Carolina, Greensboro, North Carolina \\ and \\ E. EUGENE SCHULTZ, JR. \\ California Institute of Technology, Pasadena, California
}

\begin{abstract}
If you are asked to remember an event described by a sentence, how will your memory be affected by the number of related events experienced in the same context? The experiments reported here address this empirical question within the theoretical framework of relational and itemspecific information. Assuming that both common and distinctive features of events are important in recall, encoding of both types of information should produce optimal performance. Assuming further that the type of information encoded, either common or distinctive, is influenced by manipulations, such as the number of related sentences and the orienting task, recall should be a product of the interaction between set size and type of orienting task. The results of these experiments were consistent with this prediction. Subsidiary analyses supported the interpretation of this interaction in terms of the differential availability of relational and item-specific information. The results are discussed in the context of the script pointer + tag hypothesis of schema theory.
\end{abstract}

Among the major considerations in the theoretical description of memory is the role of relational and distinctive attributes of an event. Relational attributes are those common to some or all elements of an event, whereas distinctive attributes are unique to one element of an event. Because any given event may be represented in memory by both relational and distinctive attributes, the theoretical challenge is to describe the function of relational and distinctive information in recall of that event.

For example, the organization framework for memory advocates an integrated encoding along some dimension of shared attributes. The levels of processing framework, on the other hand, has culminated in a concept of distinctiveness, emphasizing the importance of encoding unshared or distinctive features of each to-be-remembered element (Hunt \& Mitchell, 1978; Lockhart, Craik, \& Jacoby, 1976; Nelson, 1979). Thus, each of these major frameworks for memory essentially is defined by its position on the importance of relational and distinctive information; however, curiously enough, the two positions are contradictory.

Hunt and Einstein (1981), in commenting upon this inconsistency, proposed a compromise in which both common and distinctive features are assumed to be important:

Support for this research was provided by the Graduate Research Council of the University of North Carolina, Greensboro. Helpful comments on an earlier version of the manuscript were provided by Art Graesser, Cheryl Logan, and Marc Marschark. Requests for reprints should be addressed to the senior author at Department of Psychology, University of North Carolina-Greensboro, Greensboro, NC 27412. both common and distinctive components of a to-beremembered element may be encoded, and the two function differently in retrieval. For example, common information may correspond to feature overlap among elements of an event, and distinctiveness may correspond to nonoverlapping features (Tversky, 1977). Furthermore, as Tversky suggested, attention may be directed to either shared or distinctive features. Therefore, the information encoded about a set of discrete elements may include shared features, unshared features, or both. At the time of retrieval, the shared features, which represent a "category," function to delimit the search set or class from which the specific item will be drawn. Precise reconstruction of the specific item depends, however, upon activation of distinctive or unshared features.

The compromise framework outlined above has certain established empirical implications. For example, manipulations designed to encourage encoding of both shared and item-specific information produce better free recall than do manipulations that encourage encoding of only one feature (Einstein \& Hunt, 1980; Hunt \& Einstein, 1981). At the same time, the relational and item-specific tasks differentially affect other dependent measures, such as recognition, clustering, category recall, and items per category recalled. The latter findings provide support for the argument for the independence of the two types of information. Equally important, the framework is supported by empirical success in application to a variety of existing problems (Begg, 1978; Bellezza, Cheesman, \& Reddy, 1977; Einstein, McDaniel, Bowers, \& Stevens, 1984; Epstein, Phillips, \& Johnson, 1975; Glenberg, 
1979; Glenberg \& Smith, 1981; Harris, Begg, \& Upfold, 1980; Hunt \& Mitchell, 1982; Mandler, Goodman, \& Wilkes-Gibbs, 1982; Ritchey, 1980).

Most recently, Hunt and Seta (1984) demonstrated that set size effects in recall can be understood as a function of shared and item-specific information. Hunt and Seta assumed that set size reflects the number of events sharing features. They then varied the number of instances of natural categories in a list. Assuming that large categories encourage the encoding of shared information and that small categories encourage item-specific processing, Hunt and Seta felt that the addition of item-specific information to large categories and shared information to small categories should enhance recall. Indeed, their results showed higher recall of large categories following an item-specific orienting task and higher recall of small categories following a relational orienting task. The interaction between category size and orienting task was predicted from the assumption that optimal recall involves both shared and item-specific information.

The purpose of the present experiments was to extend Hunt and Seta's (1984) findings to textual materials. Beyond the obvious question of the empirical generality from word lists, the present experiments provide data relevant to conceptualizations of memory for connected discourse. Such conceptualizations, particularly schema theories (e.g., Rumelhart \& Ortony, 1977; Schank \& Abelson, 1977; Thorndyke \& Hayes-Roth, 1979), emphasize the importance of thematic or shared information. However, Einstein et al. (1984) recently demonstrated that recall of textual materials is a function of variables affecting the encoding of both shared and item-specific information.

In their second experiment, Einstein et al. (1984) presented subjects with either an ambiguous passage or a highly structured passage. Incidental orienting tasks focused attention either on the relationships among the sentences of the passage or on the individual sentences of the passage. Subsequent recall of the passage was a function of the interaction between orienting task and passage structure, such that ambiguous passages were better recalled following the relational task and structured passages were better recalled following the sentence-specific task. Einstein et al. (1984) interpreted these results as indicative of the influence of materials and orienting tasks upon the encoding of shared and distinctive information. Structured passages and relational orienting tasks encourage encoding of information shared by sentences, whereas sentence-specific orienting tasks and ambiguous passages require attention to individual sentences. Because recall of the sentences theoretically requires the availability of both shared and distinctive information, superior performance is predicted for conditions involving nonredundant combinations of passages and orienting tasks.

The presert study provides additional evidence on this question. In these experiments, subjects read sentences describing events related to a central theme. Each of three lists contained sentences related to a different theme, and the number of sentences related to each theme varied. Sub- jects were asked to perform orienting tasks that were designed to draw their attention either to the relationships among the sentences or to each individual sentence. If both shared and item-specific information are important to memory for specific sentences, subjects' performance should be best following relational orientation to small sets and item-specific orientation to large sets; that is, a large number of sentences related to a theme draws attention to features shared by the sentences and away from distinctive attributes of each sentence. Hence, orientation to individual sentences in large sets would supplement the shared information with sentence-specific information. Likewise, small sets are likely not to be encoded relationally, but to be encoded as individual items. Relational orientation to these small sets, therefore, should supplement the encoding of sentence-specific information and improve performance.

Further support for this analysis is provided by indices of thematic and sentence-specific information which are assessed independently of total recall. The theoretical framework motivating these experiments specifies that measures of shared and item-specific information should be sensitive to the orienting tasks and set size in ways that occasionally are different from recall. For example, large sets and relational orientation should produce very high recall of theme, but relatively poor recall of sentences, due to the lack of sentence-specific information. Thematic information is indexed by recall of at least one sentence from a given theme. Sentence-specific information is indexed by the proportion of sentences recalled given access to a particular theme. The rationale for this measure is that within-theme recall requires information beyond that shared by the instances of the theme. In summary, the predicted interaction between orienting task and set size upon recall is further analyzed in terms of shared and item-specific information as indexed by recall of themes and sentences per theme.

\section{EXPERIMENT 1}

\section{Method}

Design and Subjects. In Experiment 1, set size was manipulated such that each subject received a list of 14 sentences. Within the list, 8 sentences were related to one theme, 4 related to a second theme, and 2 related to a third theme. The subjects performed one of two orienting activities on this list, either a thematic sorting or pleasantness rating.

The subjects were 71 volunteers from introductory psychology, 35 of whom were assigned to the pleasantness-rating condition and 36 to the category-sorting condition

Materials. The sentences used in the experiment were simple one- or two-proposition descriptions of events that might occur at either a circus, an airport, or a football game. Eight different sentences were written for each theme (see Table 1).

The sentences were typed individually on index cards, and each subject saw a list of 14 sentences. Sentences from a given theme appeared equally often for each set size, and within this counterbalancing, 12 separate lists were constructed such that each sentence appeared equally often across all subjects. For example, a given list had 8 sentences from the circus theme, 4 from the football theme, and 2 from the airport theme. Four separate versions 
Table 1

Themes and Sentences Used in Both Experiments

Plane Trip

The cabbie drove to the airport.

The pilot checked the instruments.

The passengers buckled their seatbelts.

The controller cleared the runway.

The bystanders watched the take-off

The no-smoking sign was flashing.

The stewardess offered a beverage.

The plane landed at its destination.

Football Game

The score was 14 to 7 .

The stadium was packed.

The cheerleader turned a cartwheel.

The player threw a pass.

The referee called a penalty.

The team piayed good defense.

The band played at half-time.

The crowd cheered the touchdown.

Circus

The elephants paraded around the ring

The performer walked the tightrope.

The clown wore baggy pants.

The trainer cracked his whip.

The cages contained wild animals.

The ringmaster announced the acts.

The acrobat swung on the trapeze.

The monkeys were dressed in fancy clothes.

of this list were then prepared, so that each sentence from the airport theme would be represented in the data. Finally, the order of each list was random, with the restriction that no more than 2 sentences from a given theme occurred contiguously.

Procedure. The experiment was conducted under conditions of incidental memory in which the subjects were told that they were providing normative data on the sentences. Sorting instructions required subjects to place each of the 14 cards beneath its appropriate category heading. The cards were to be placed facedown, and the thematic headings remained in view at all times. For the pleasantness rating, subjects were instructed to examine each sentence carefully and to rate its pleasantness on a 5-point scale, where 1 = very pleasant and $5=$ very unpleasant. Upon completion of the orienting tasks, subjects were given standard free-recall instructions and were allowed $4 \mathrm{~min}$ for recall.

\section{Results}

Recall was scored using both gist and verbatim criteria, but each analysis produced the same conclusions; therefore, the data reported are from the verbatim scoring. Results are presented for three separate dependent measures: free recall, theme recall, and sentences per theme recall.

Free-recall data are shown in Figure 1 as a function of orienting task and set size. The most striking aspect of Figure 1 is the interaction between set size and orienting task, $[F(2,126)=3.61, M S e=.08$ (all results reported as reliable reached at least the .05 level)]. Further analysis of this interaction via planned comparisons provided consistent, if somewhat weak, support for the proposed theoretical analysis. Recall of eight-sentence sets was marginally superior $(p<.07)$ following pleasantness rating when compared to sorting. Performance on the small (two-sentence) sets also was marginally superior $(p<.07)$ for the sorting group compared to the pleasantness-rating group. Furthermore, the sorting task led to significantly better recall of small than large categories; however, contrary to expectations, the pleasantness-rating task did not produce reliably different performance as a function of set size. In summary, recall was a function of the interaction between set size and orienting task. The interaction resulted primarily from better recall of large sets following pleasantness rating and better recall of small sets following sorting.

Overall recall was further analyzed into themes recalled and sentences per theme recalled as indices of relational and item-specific information, respectively. These measures are presented in Table 2 as a function of set size and orienting task. Consider, first, recall of themes, defined as recall of at least one sentence from the thematic set. The first two rows of Table 2 show nearly perfect recall of themes from eight-sentence sets, regardless of orienting task. However, theme recall declines with small sets and appears to be much better following sorting than following pleasantness rating for two-sentence sets. Since theme recall is based on categorical data, the results in Table 2 were subjected to Chi-square analyses comparing the two orienting tasks at each set size. In spite of the apparent interaction shown in Table 2, none of the three Chi-square comparisons was reliable.

The percentages of sentences per theme recalled are presented in the third and fourth rows of Table 2. Pleasantness rating produced better sentences per theme recall than did sorting $[F(1,69)=5.5, M S e=.04]$. Twosentence sets also produced better sentence per theme recall than did four- or eight-sentence sets $[F(2,102)=$ $8.5, M S \mathrm{e}=.04]$. As predicted, however, these effects were qualified by an interaction between set size and orienting task $[F(2,102)=35.5, M S \mathrm{e}=.04]$. Recall of

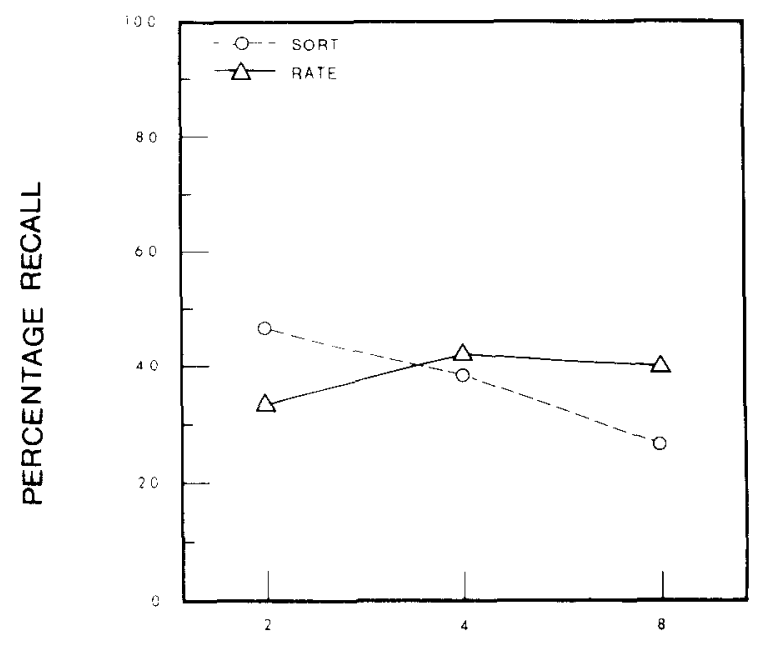

SET SIZE

Figure 1. Percentage of correct recall as a function of set size and orienting task in Experiment 1. 
Table 2

Mean Percentages of Categories and Items per Category Recalled in Experiment 1

\begin{tabular}{cccccc} 
& \multicolumn{2}{c}{ Recalled in Experiment } & \multicolumn{2}{c}{$\begin{array}{c}\text { Sentences per Theme } \\
\text { Recalled }\end{array}$} \\
\cline { 2 - 2 } \cline { 5 - 6 } Set Size & Sorting & Rating & & Sorting & Rating \\
\hline 2 & .67 & .51 & .71 & .67 \\
4 & .92 & .80 & .42 & .53 \\
8 & .97 & .97 & .28 & .41 \\
\hline
\end{tabular}

sentences per theme was best for two-sentence sets, regardless of orienting task. ${ }^{1}$ For the eight-sentence sets, pleasantness rating led to better sentences per theme recall than did sorting. Thus, in contrast to the index of relational information, the index of item-specific information showed superior performance with small sets and pleasantness-rating orientation.

\section{Discussion}

Recall of propositions was influenced by both set size and orienting task. With eight sentences pertaining to the same theme, memory was marginally better following the pleasantness-rating orienting task, but with only two sentences related to a theme, the sorting task produced slightly higher performance. Moreover, small sets were better remembered than large sets within the sorting task, and although the differences were not reliable, the trend within the pleasantness-rating task was toward better memory for large sets.

Decomposition of the recall scores into recall of themes and sentences per theme suggests that the interaction in recall was due to differential availability of thematic and sentence-specific information. Theme recall of eightsentence sets was nearly perfect, regardless of orienting task, but declined markedly for two-sentence sets. Contrary to expectations, however, sorting did not produce significantly higher theme recall than did pleasantness rating with two-sentence sets. In contrast, the proportion of sentences per theme recalled was best with the twosentence sets and was inversely related to set size. Moreover, with the eight-sentence sets, the pleasantnessrating task produced better sentences per theme recall than did the sorting task. These differential effects of set size and orienting task upon theme and sentences per theme recall are important for the argument that these measures are indices of different types of information.

Although the pattern of results generally was consistent with expectations derived from the individual itemrelational processing framework, several important predictions were only marginally supported. In particular, pleasantness rating produced slightly higher recall of large sets than did sorting, and sorting led to slightly higher recall of small sets than did pleasantness rating. Furthermore, recall was not affected by set size within the pleasantness-rating task, and orienting tasks did not affect theme recall of small sets. All of the comparisons were highly reliable in the Hunt and Seta (1984) study and should have emerged here.
Each of these cases involves the pleasantness-rating task, and it is conceivable that rating the pleasantness of sentences engenders considerable relational processing. That is, the pleasantness rating of a sentence presumably is made on the basis of the sentence's meaning. Activation of the meaning of individual sentences theoretically entails the activation of shared features for related sentences. Thus, the pleasantness-rating task may have produced substantial encoding of relational information; if so, the marginal significance of the four important comparisons mentioned above would be explained. To explore this possibility and the generality of the other predicted outcomes of Experiment 1, the individual item-orienting task was changed for Experiment 2 . Instead of rating the pleasantness of sentences, subjects were required to fill in missing letters of words in the sentences. Einstein et al. (1984) showed that this task focuses attention upon individual sentences rather than on relationships among sentences. Therefore, the data from Experiment 2 should replicate those of Experiment 1, except in cases in which individual item information serves an important function.

\section{EXPERIMENT 2}

\section{Method}

Experiment 2 replicated Experiment 1 in all details except the item-specific orienting task. Rather than making a pleasantness rating, subjects were asked to supply missing letters for words in each of the sentences. Approximately $25 \%$ of the letters were missing from each sentence; this is equal to roughly one or two missing letters from each word. All subjects performing the blanks task successfully completed all of the sentences. A total of 68 subjects participated in the experiment, equally divided between the two orienting tasks.

\section{Results and Discussion}

As in Experiment 1, the data were scored according to both gist and verbatim criteria, but because the statistical conclusions were the same, only the verbatim scoring is reported. Again, the results are discussed in terms of three dependent variables: free recall, theme recall, and sentences per theme recalled.

Free-recall performance is depicted in Figure 2 as a function of set size and orienting task. The most striking aspect of Figure 2 is the interactive effect of set size and orienting task upon memory $[F(2,120)=8.32$, $M S e=.07]$. Analysis of the interaction revealed better recall of small sets following sorting than following the blanks task, and superior recall of large sets following the blanks task. Furthermore, the sorting task produced significantly higher recall of small sets than large sets. Contrariwise, the blanks task produced higher recall of large sets than small sets. Thus, as in Experiment 1, the interaction between set size and orienting task was due to superior performance on large sets following an itemspecific task and on small sets following a relational task.

The percentages of themes and sentences per theme recalled are presented in Table 3 . Theme recall, defined as recall of at least one sentence from a thematic set, was 


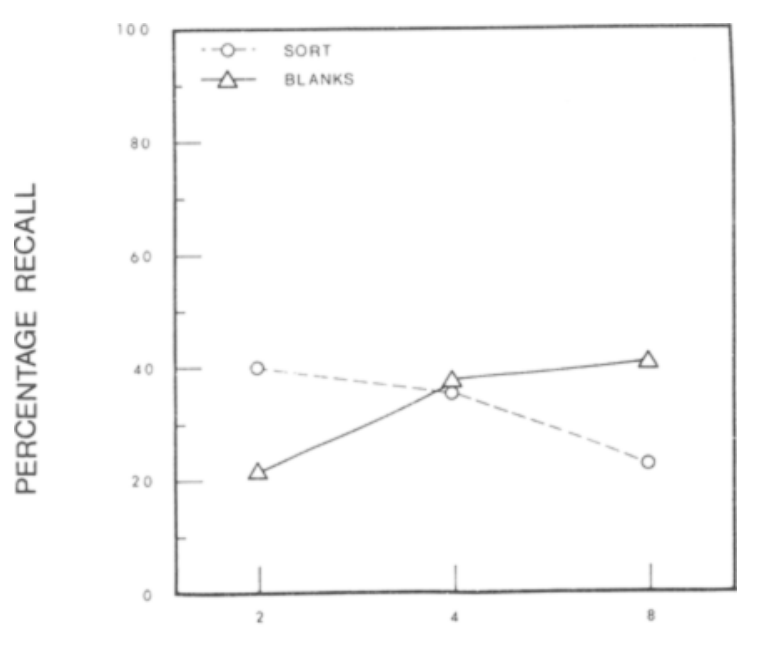

SET SIZE

Figure 2. Percentage of correct recall as a function of set size and orienting task in Experiment 2.

quite high for large sets and declined with small sets. As in Experiment 1, these data were analyzed via Chi-square comparisons between orienting tasks at each set size. Theme recall did not differ between sorting and blanks tasks at Set Sizes 8 and 4. At Set Size 2, however, sorting produced significantly higher recall than did the blanks task $\left(\chi^{2}=4.61\right)$.

Analysis of the sentences per theme recall revealed a reliable effect of set size $[F(2,92)=23.74$, MSe $=.03]$. This effect is qualified, however, by a reliable interaction between set size and orienting task $[F(2,92)=4.97$, $M S \mathrm{e}=.03 \mathrm{~J}$. Subsequent Newman-Keuls analysis of this interaction showed a reliable superiority of blanks task over the sorting task at Set Size 8, but no difference between the two tasks at Set Sizes 2 and 4.

\section{GENERAL DISCUSSION}

The results of Experiment 2 replicate and refine the results of Experiment 1. Recall was best for large sets following the blanks task and for small sets following the sorting task. Unlike the pleasantness-rating task in Experiment 1 , the blanks task produced reliably higher recall of large sets than did sorting, and sorting produced reliably higher recall of small sets than did the blanks task. Furthermore, recall of large sets was reliably higher than

Table 3

Mean Percentages of Categories and Items per Category Recalled in Experiment 2

\begin{tabular}{cccccc} 
& \multicolumn{2}{c}{ Recalled in Experiment 2 } \\
& \multicolumn{2}{c}{ Themes Recalled } & & \multicolumn{2}{c}{$\begin{array}{c}\text { Sentences per Theme } \\
\text { Recalled }\end{array}$} \\
\cline { 2 - 3 } \cline { 5 - 6 } Set Size & Sorting & Blanks & & Sorting & Blanks \\
\hline 2 & .67 & .41 & & .60 & .54 \\
4 & .75 & .87 & & .48 & .43 \\
8 & .86 & .97 & & .27 & .42 \\
\hline
\end{tabular}

that of small sets following the blanks task, and finally, theme recall was significantly greater for the sorting condition than the blanks condition at the two-sentence set size. Each of these predicted results, which were not reliable in Experiment 1, emerged with the change to the blanks task in Experiment 2.

In general, the results of the two experiments demonstrate that memory for specific sentences is best when conditions leading to the processing of the two different types of information are combined. Assuming that both shared and item-specific information are important in recall, and that large sets and sorting tasks encourage relational encoding whereas small sets and blanks or pleasantnessrating tasks encourage item-specific encoding, the pattern of results obtained in these experiments is easily understood. These two variables presumably influence the attended and encoded information, and thus, the data suggest that recall of specific sentences is optimal when both shared and item-specific information is encoded. At a general level, these experiments, along with Einstein et al. (1984), extend the analysis of memory in terms of shared and item-specific information (Humphreys \& Bain, 1983) to recall of units other than single words. More specifically, the data replicate the results, and extend the interpretation, of Hunt and Seta's (1984) analysis of set size effects upon recall.

The proposed framework assumes the importance of both shared and item-specific information in recall. Shared information functions in retrieval to delineate a class of events, whereas item-specific information provides precise access to a specific event within the class. Encoding of the two types of information may be influenced by a variety of factors, including the person's intentions, modeled here by orienting tasks, and characteristics of the material itself, as in the present case, the number of sentences related to a given theme. If very few related sentences are present, the individual may comprehend each separate sentence, thereby obtaining item-specific information, but simultaneously fail to detect the relationships among sentences. A large number of related sentences, however, draws attention to the shared features of the sentences at the expense of attention to their differences.

As noted by Einstein et al. (1984), schema-based theories of text processing (e.g., Anderson \& Pickert, 1978; Rumelhart \& Ortony, 1977; Schank \& Abelson, 1977) are primarily concerned with the role of shared information in comprehension and memory. Schema may serve to focus encoding and provide a top-down retrieval plan, which accesses specific propositions as functions of their centrality to the schema (Yekovich \& Thorndyke, 1981); this is precisely the role of shared information.

In agreement with the conclusions of Einstein et al. (1984), however, our data seem to require a role for proposition-specific processing in encoding and retrieval. As in the experiment of Einstein et al., situations optimal for encoding shared information (large sets and sorting task in the present case) do not produce optimal recall of specific propositions. One version of schema theory 
that includes a "script pointer plus tag" ( $\mathrm{SP}+\mathrm{T})$ hypothesis (Graesser, Woll, Kowalski, \& Smith, 1980; Schank \& Abelson, 1977) offers the possibility of an important conceptual role for item-specific information. In this view, general scripts are activated at encoding, and specific actions within that script are tagged. The script relates the specific actions and, in that sense, represents shared information, and tagged actions correspond to item-specific information. If set size is assumed to affect activation of the appropriate script and the orienting tasks are assumed to affect the probability or strength of "action-tagging," the account of the SP+T hypothesis of encoding is identical to the present description.

At retrieval, however, the two ideas seem to differ in an important respect. According to the SP+T hypothesis, retrieval in recall is guided solely by the script or shared information and is not determined by item-specific information (Graesser et al., 1980, p. 513). Such a description provides a satisfactory account of much of the literature on memory for text in which recall of specific ideas may be poor. It is well established, however, that precision in recall of connected discourse can be quite good. Thus, a more complete account should include some function for the tagged action at retrieval. This function would be discriminative and served by item-specific information; indeed, such a function is proposed for tagged actions in recognition memory by Graesser et al.

The point of this discussion and the relevance of our data to schema theory are not to suggest that the concept of script be replaced with relational and item-specific processing. In some sense, the ideas are at different levels of generality and serve different purposes. Rather, the suggestion is that memory for specific events can be conceptualized as being a function of shared and item-specific information, and particular theories of memory should include roles for both types of information.

\section{REFERENCES}

Anderson, R. C., \& Pickert, J. W. (1978). Recall of previously unrecallable information following a shift in perspective. Joumal of Verbal Learning \& Verbal Behavior, 17, 1-12.

BEGG, I. (1978). Similarity and contrast in memory for relations. Memory \& Cognition, 6, 509-517.

Bellezza, F. S., Cheesman, F. L., \& Reddy, G. (1977). Organizational and semantic elaboration in free recall. Journal of Experimental Psychology: Human Learning \& Memory, 1, 539-550.

EINSTEIN, G. O., \& HUNT, R. R. (1980). Levels of processing and organization: Additive effects of individual-item and relational processing. Joumal of Experimental Psychology: Human Learning \& Memory, 6, 588-598.

Einstein, G. O., McDaniel, M. A., Bowers, C. A., \& Stevens, D. T. (1984). Memory for prose: The influence of relational and propositionspecific processing. Journal of Experimental Psychology: Learning, Memory, \& Cognition, 10, 133-143.

Epstein, M. L., Phillips, W. D., \& Johnson, J. J. (1975). Recall of related and nonrelated word pairs as a function of processing level. Journal of Experimental Psychology: Human Learning \& Memory, 1, 149-152.

Glenberg, A. M. (1979). Component levels theory of the effects of spacing of repetitions on recall and recognition. Memory \& Cognition, 7, 95-112.

Glenberg, A. M., \& Smith, S. M. (1981). Spacing repetitions and solving problems are not the same. Journal of Verbal Learning \& Verbal Behavior, 20, 110-119.

Graesser, A. C., Woll, S. B., Kowalski, D. J., \& Smith, D. A. (1980). Memory for typical and atypical actions in scripted activities. Journal of Experimental Psychology: Human Learning \& Memory, 6, 503-515.

HARRIS, G., BEGG, I., \& UPFOLD, D. (1980). On the role of the speaker's expectations in interpersonal communication. Journal of Verbal Learning \& Verbal Behavior, 19, 597-607.

HUMPhREYS, M. S., \& BAIN, J. D. (1983). Recognition memory: A cue and information analysis. Memory \& Cognition, 11, 586-600.

Hunt, R. R., \& Einstein, G. O. (1981). Relational and item-specific information in memory. Journal of Verbal Learning \& Verbal Behavior, 20, 497-514.

HunT, R. R., \& MitchelL, D. B. (1978). Specificity in nonsemantic orienting tasks and distinctive memory traces. Journal of Experimental Psychology: Human Learning \& Memory, 4, 472-481.

Hunt, R. R., \& Mitchell, D. B. (1982). Independent effects of semantic and nonsemantic distinctiveness. Journal of Experimental Psychology: Learning, Memory \& Cognition, 8, 81-87.

Hunt, R. R., \& SETA, C. E. (1984). Category size effects in recall: The roles of relational and individual item information. Journal of Experimental Psychology: Learning, Memory \& Cognition, 10, 454-464.

Lockhart, R. S., Craik, F. I. M., \& Jacoby, L. L. (1976). Depth of processing, recognition and recall. In J. Brown (Ed.), Recognition and recall. London: Wiley.

Mandler, G., Goodman, G. O., \& Wilkes-Gibis, D. L. (1982). The word frequency paradox in recognition. Memory \& Cognition, 10, 33-42.

Nelson, D. L. (1979). Remembering pictures and words: Appearance, significance, and name. In L. S. Cermak \& F. M. Craik (Eds.), Levels of processing in human memory. Hillsdale, $\mathrm{NJ}$ : Erlbaum.

RrTchey, G. H. (1980). Picture superiority in free recall: The effects of organization and elaboration. Journal of Experimental Child Psychology, 29, 460-474.

Rumelhart, D. E., \& Ortony, A. (1977). The representation of knowledge in memory. In R. C. Anderson, R. J. Spiro, \& W. E. Montague (Eds.), Schooling and the acquisition of knowledge. Hillsdale, NJ: Erlbaum.

SChANK, R. C., \& ABELSON, R. (1977). Scripts, plans, goals, and understanding. Hillsdale, NJ: Erlbaum.

ThORNDYKE, P. W., \& HAYEs-RoTH, B. (1979). The use of schemata in the acquisition and transfer of knowledge. Cognitive Psychology, 11, 82-106.

TVersky, A. (1977). Features of similarity. Psychological Review, 84, 327-352.

Yekovich, F. R., \& Thorndyke, P. W. (1981). An evaluation of alternative functional models of narrative schemata. Journal of Verbal Learning \& Verbal Behavior, 20, 454-469.

\section{NOTE}

1. Ian Begg and Don Homa have previously pointed out to us that the sentence per theme comparison has the potential difficulty of different mathematical ranges for different set sizes. The minimum percentage possible for an eight-sentence set is .125 , whereas the minimum for a two-sentence set is . 50 . Note first that this is a mathematical, not psychological, restriction. More importantly, this is not an issue in comparisons between orienting tasks at the same set size or in the interaction which is the most important aspect of the data.

(Manuscript received March 22, 1985; revision accepted for publication July 22,1985 .) 